Journal of

Synchrotron

Radiation

ISSN 0909-0495

Received 15 April 2005

Accepted 20 July 2005

(C) 2005 International Union of Crystallography

Printed in Great Britain - all rights reserved

\section{Structures from powders and poor-quality single crystals at high pressure}

\author{
Malcolm I. McMahon
}

School of Physics and Centre for Science at Extreme Conditions, The University of Edinburgh, Kings Buildings, Mayfield Road, Edinburgh, UK. E-mail: mim@ph.ed.ac.uk

The use of single-crystal techniques and quasi-single-crystal samples in solving and refining complex crystal structures at high pressure is reviewed. In particular, recent studies of the incommensurate and modulated structures found in a number of elemental metals at high pressure are focused on.

Keywords: high-pressure; single crystal; incommensurate.

\section{Introduction}

The early 1990s saw great advances in the determination of crystal structures at high pressures. The development of angledispersive diffraction techniques using imaging plates in Japan (Shimomura et al., 1992), their subsequent development by our group at the Synchrotron Radiation Source (SRS) at Daresbury Laboratory in the UK (Nelmes \& McMahon, 1994), and their utilization at other synchrotron sources around the world has led to structural studies of unprecedented detail (Nelmes \& McMahon, 1998; Paszkowicz, 2002). By the end of the 1990s, however, it was clear that in an increasing number of cases the structures being revealed by powder studies were of such complexity that the inherent limitations of the powder method were becoming a limiting factor in their study: powder-diffraction data are inherently one-dimensional; peaks with similar $d$-spacings overlap; only a small number of crystallites in a powder sample actually diffract if the sample is kept stationary; and the distribution of the crystallite orientations in a powder sample at high pressure is typically non-random. While some of these problems can be overcome to some extent by using higher-resolution detectors or by using larger samples that are rotated in the X-ray beam (Wright et al., 1996; Belmonte, 1998), the one-dimensional nature of the powder data means that indexing unknown powder profiles, which is the first step in solving any unknown structure, remains extremely difficult. This is particularly true in the case of incommensurate structures, where the standard indexing software will very rarely, if at all, be able to determine the unit cell.

It was clear, therefore, that further advances in structure solution and refinement at high pressure could only occur through the use of single-crystal techniques. A key and timely development in this field was the widespread appearance of CCD-equipped diffractometers in the late 1990s. These instruments have revolutionized the field of structural biology, and also provide many benefits for the study of single crystals at high-pressure. CCD detectors allow many reflections to be measured simultaneously, reducing data collection times from days to hours; the unit cell and crystal orientation need not be known before data is collected; sections of the reciprocal lattice can be reconstructed from the CCD images, enabling studies of diffuse scattering and anisotropic peak shapes; and if there is more than one crystal in your sample, data can be collected from both of them simultaneously. However, it is not always possible to obtain a single crystal of a high-pressure phase and, because hundreds of diffraction images need to be collected as the single crystal is rotated in the X-ray beam, data collection from single crystals is considerably slower than when using powder methods even when using a CCD detector. But the advantages are typically so great that single-crystal techniques should be used whenever possible. Furthermore, modern synchrotron sources and area detectors enable singlecrystal techniques to be used on samples where this was not possible before. Any form of poor quality single crystal, or quasi-single-crystal, from which it is possible to obtain a unit cell and orientation matrix can be used to identify each and every feature in the diffraction pattern from both the (quasi-)single-crystal and, if powder data is available from the same sample, from this data too.

\section{Experimental details}

All the diffraction data illustrated in this paper were collected on stations 9.1 and 9.8 at the Synchrotron Radiation Source (SRS) at Daresbury Laboratory or on in-house facilities. In all cases powder and single-crystal data were collected using angle-dispersive techniques with a wavelength of $\sim 0.46 \AA$ or $\sim 0.68 \AA$ (at SRS) or $0.71 \AA$ (in-house). The two-dimensional Debye-Scherrer patterns from powder samples were integrated azimuthally and then refined using Rietveld methods (Nelmes \& McMahon, 1994). Single-crystal data were obtained using either a Bruker SMART 1K CCD detector (SRS) or a Bruker APEX CCD detector (in-house). All samples were compressed in diamond anvil pressure cells, and the pressure was determined using the ruby fluorescence method. Full details of the techniques used are given by Nelmes \& McMahon (1994) and in the papers describing the 
individual studies. A review of the structural details of all the incommensurate structures found in the elements at high pressure has been published recently (McMahon \& Nelmes, 2004).

\section{Results}

As an example of what is now possible with a quasi-singlecrystal, Fig. 1 shows a diffraction pattern obtained from a quasi-single-crystal of Rb-III at 14.3 GPa (Nelmes et al., 2002). The sample comprises seven different crystallites, as shown in the inset and, as a result, the diffraction peaks have a width in $\omega$ of $\sim 15^{\circ}$. Despite the extreme widths of these peaks, it was possible to collect a full single-crystal data set from this sample by ensuring that the integration 'box' around each Bragg peak was large enough $\left(\sim 18^{\circ}\right.$ in $\left.\omega\right)$ to include the intensity from all seven crystallites. The resulting data set of 470 unique reflections was then solved straightforwardly using direct methods (Nelmes et al., 2002).

In addition to using standard single-crystal methods, we have found that a combination of powder and single-crystal methods can be extremely powerful in determining unknown crystal structures. As an example of using a combination of single-crystal and powder data, Fig. 2 shows the diffraction profiles from (a) a quasi-single-crystal of Bi-III at $4.5 \mathrm{GPa}$ and (b) a powdered sample of Bi-III at the same pressure. The single-crystal image reveals diffuse scattering and two classes of reflections: those lying on the planes of diffuse scattering and those that are not. Neither the diffuse scattering nor the two classes of reflection are discernible in the two-dimensional powder pattern. Using the two patterns it was possible to match the $2 \theta$ values of each Bragg reflection in pattern $(a)$ with its corresponding diffraction ring in $(b)$, and thus sort the powder pattern into the two different classes of reflection. It

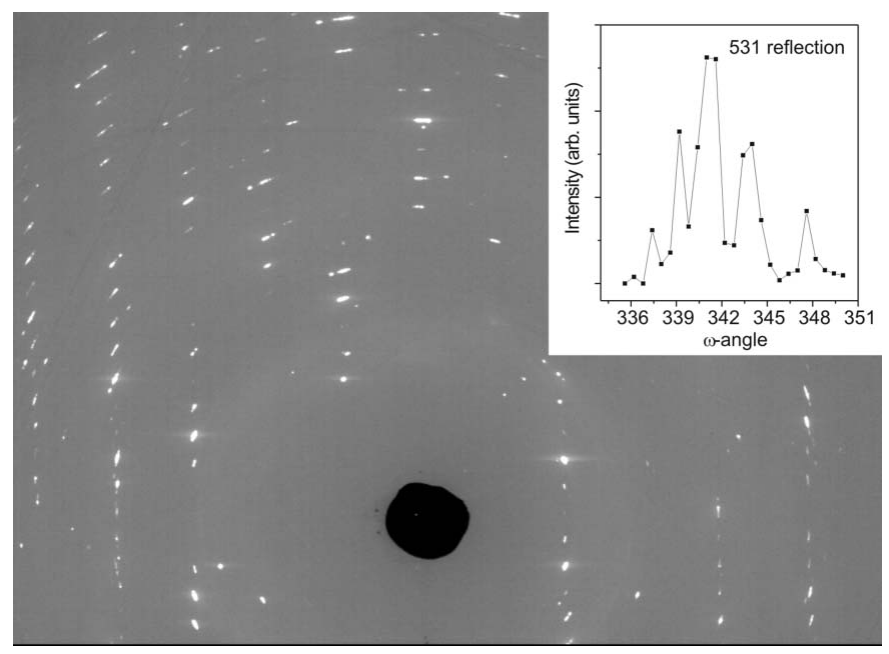

Figure 1

Diffraction pattern from a poor-quality single crystal of Rb-III at 14.3 GPa. Layer lines of reflections are clearly visible. The inset shows a scan through the (531) reflection in a direction approximately perpendicular to the plane of the image. The broader features at $341^{\circ}$ and $344^{\circ}$ each comprise two almost overlapping peaks. After Nelmes et al. (2002). was then straightforward to index the two classes as being from a body-centred host structure with a tetragonal unit cell with $a_{\mathrm{H}}=8.602$ (1) $\AA$ and $c_{\mathrm{H}}=4.207$ (1) $\AA$ and a body-centred tetragonal guest structure with a unit cell $a_{\mathrm{G}}=8.602$ (1) $\AA$ and $c_{\mathrm{G}}=3.211$ (1) $\AA$ (McMahon, Degtyareva \& Nelmes, 2000). Bi-III thus has an incommensurate composite structure, a $c$ axis projection of which is shown in Fig. 3. Similar incommensurate structures have since been found in the other group $\mathrm{V}$ elements Sb and As (Schwarz et al., 2003; Degtyareva et al., 2004a,b), and also in $\mathrm{Ba}, \mathrm{Sr}$ and $\mathrm{Rb}$ (Nelmes et al., 1999; McMahon, Bovornratanaraks et al., 2000; McMahon et al.,
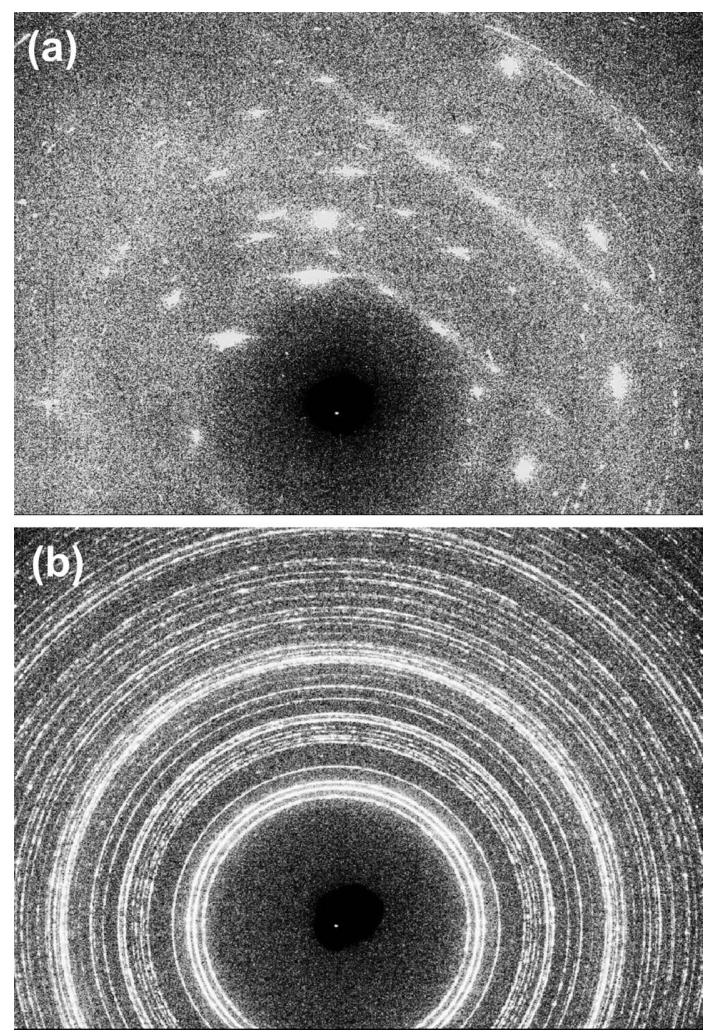

Figure 2

Two-dimensional diffraction patterns from $(a)$ a quasi-single-crystal of BiIII at $4.5 \mathrm{GPa}$, and $(b)$ a powder of Bi-III at the same pressure. The sheets of diffuse scattering are seen edge-on in image $(a)$.

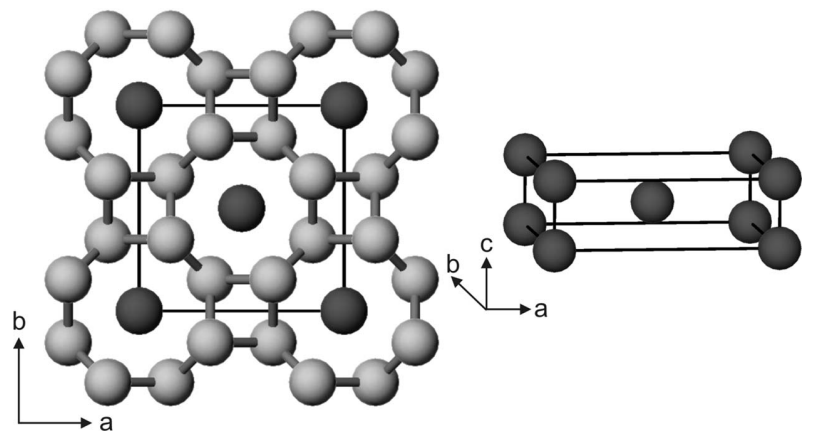

Figure 3

The incommensurate composite structure of Bi-III shown in projection down the $c$-axis. The host atoms located at $z=0$ and $z=1 / 2$ are shown using light grey shading, and the guest atom chains are shown in dark grey. The inset shows a perspective drawing of the tetragonal guest structure. 
2001). Note that, in the indexing of the powder profile in Fig. 2(b), no actual analysis of the single-crystal data was performed other than determining the $2 \theta$ values of the two classes of reflections from a single two-dimensional image.

We used a similar technique in our recent solution of the long-uncertain structure of Te-III, reported to be stable between 7 and $11 \mathrm{GPa}$. Despite their extremely high quality, we were initially unable to index the powder diffraction profiles from Te-III. However, patterns collected as a function of pressure revealed that they contained two classes of reflection: intense peaks which coalesced at higher pressures, and weaker peaks which became weaker still at higher pressures but remained as singlets. Indexing only the first class of reflections revealed that they could all be indexed on a bodycentred monoclinic (b.c.m.) cell with $a=3.9181$ (1), $b=$ $4.7333(1), c=3.0612(1) \AA$ and $\beta=113.542(2)^{\circ}$ at $8.5 \mathrm{GPa}$ (Hejny \& McMahon, 2003). However, this unit cell, and closely related cells or supercells, were unable to account for all the weaker peaks in the profiles. After almost 30 attempts, however, we were able to produce a twinned quasi-singlecrystal of Te-III, a diffraction image from which is shown in Fig. 4. This revealed immediately that the peaks that were not fitted by the b.c.m. cell were all satellite peaks arising from an incommensurate $q$-vector $(0,0.288,0)$ at $8.5 \mathrm{GPa}$, and that TeIII thus has a modulated structure (Hejny \& McMahon, 2003). Once this had been determined from the single-crystal data, it was possible to refine the modulated structure of Te-III as a function of pressure from the powder profiles. The same modulated structure has since been observed at higher pressures in both Se-IV and S-III from data collected to 70 and $100 \mathrm{GPa}$, respectively, on station ID09 at the European Synchrotron Radiation Facility in Grenoble (McMahon et al., 2004; Hejny et al., 2005). As in the case of Bi-III above, we could only have solved Te-III, and hence Se-IV and S-III, with the aid of single-crystal data, and, as with Bi-III, we did not need to determine the intensities of the peaks from the singlecrystal sample of Te-III. Only the orientation matrix of the

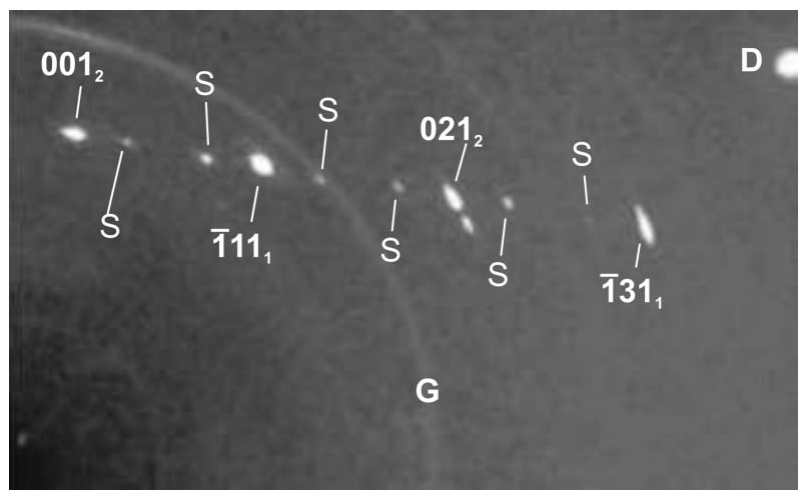

Figure 4

Part of the single-crystal diffraction pattern from Te-III at 7.4 GPa. The incommensurate satellite peaks are marked with an $\mathrm{S}$. The main reflections from the two twin components are indexed $h k l_{1}$ and $h k l_{2}$. Diffraction features from one of the diamond anvils and the metal gasket are labelled D and G, respectively. Modified after Hejny \& McMahon (2004). sample was determined, using the unit cell obtained initially from the powder data, from which it was then possible to index the satellite reflections arising from the modulations of the structure.

A final example demonstrating the power of combined single-crystal and powder methods is the solution of the structure of Te-II, the phase of tellurium stable between 4 and $7 \mathrm{GPa}$. The very large number of diffraction peaks visible in powder profiles from Te-II (Fig. 5) suggested that the structure was considerably more complex than that of Te-III. However, attempts to index the Te-II powder profiles using standard software were unsuccessful and so we resorted to a singlecrystal study using the same crystal we had used for the solution of Te-III (Hejny \& McMahon, 2003). Diffraction images obtained from this sample as the pressure was reduced from 7.4 to $3.9 \mathrm{GPa}$ are shown in Fig. 6. The sample was initially Te-III (Fig. 6a), but gradually transformed to a mixture of Te-III and Te-II (Fig. $6 b$ and $6 c$ ) before transforming to a quasi-single-crystal of Te-II (Fig. $6 d$ ).

After locating all of the observable diffraction peaks by hand, analysis of the positions and splitting of the diffraction peaks in Te-II at $3.9 \mathrm{GPa}$ (Fig. $6 d$ ) relative to those in Te-III at 7.4 GPa (Fig. 6a) showed that they are consistent with both the $\alpha$ and $\gamma$ angles of the monoclinic Te-III structure deviating slightly from $90^{\circ}$ in Te-II. Te-II is thus triclinic. Furthermore, the Te-II peaks that arise from the splitting of the Te-III satellite peaks indicate that the $b$ lattice parameter of Te-II is three times longer than that of Te-III. Te-II thus has a very close relationship to the (commensurate) Te-III structure obtained when the $q$ vector is $(0,1 / 3,0)$ and thus when the $b$ axis of Te-III is tripled in length. This close similarity between Te-II and Te-III also enabled an initial structural model for Te-II to be made, and the full Rietveld refinement of the triclinic structure is shown in Fig. 7 (Hejny \& McMahon, 2004). Subsequent studies of Se and S at SRS and ESRF have shown that the same triclinic structure is found there too (Hejny \& McMahon, 2004; Hejny et al., 2005). Once again, the solution to these long unknown structures would not have been possible without the use of single-crystal techniques.



Figure 5

Diffraction profile from Te-II at $4.9 \mathrm{GPa}$, showing the complexity of the diffraction pattern. The inset shows the single very weak reflection at a $d$ spacing of $\sim 7.1 \AA\left(2 \theta=3.8^{\circ}\right)$. 


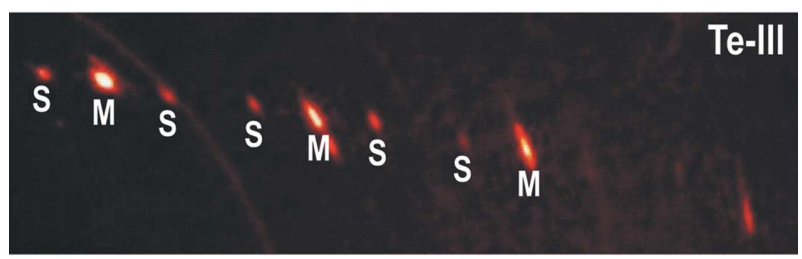

(a)

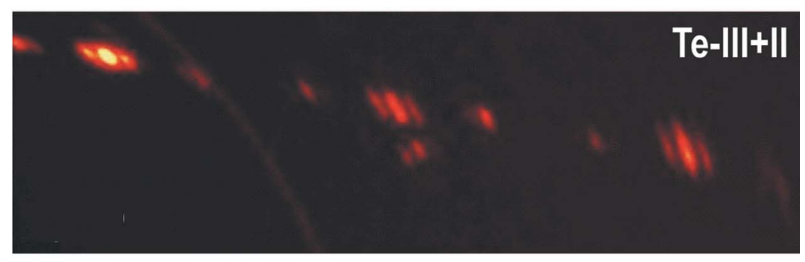

(b)

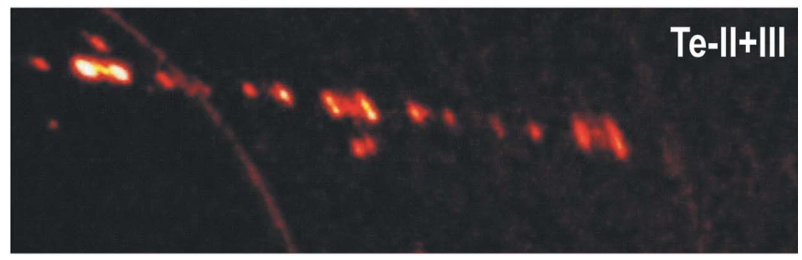

(c)

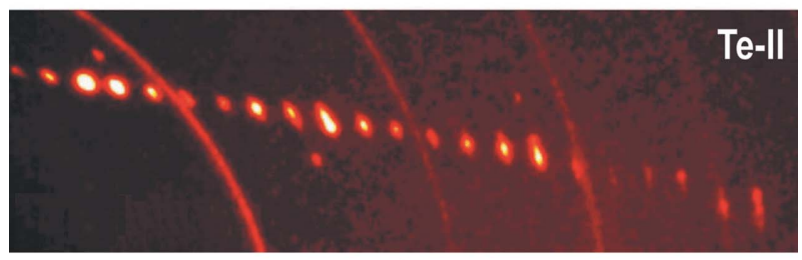

(d)

Figure 6

Single-crystal diffraction images of Te collected on pressure decrease from (a) pure Te-III at 7.4 GPa, showing the main (M) and satellite (S) reflections, $(b)$ mixed Te-III/Te-II at $5.0 \mathrm{GPa},(c)$ mixed Te-II/Te-III at 4.2 $\mathrm{GPa}$, and $(d)$ almost single-phase Te-II at 3.9 GPa. After Hejny \& McMahon (2004).

\section{Conclusions}

Modern CCD-equipped diffractometers have made possible high-pressure single-crystal studies that were previously either too difficult or too time-consuming to perform with point detectors. In particular, a combination of CCD detectors and high-intensity synchrotron radiation means that even very poor quality single crystals can yield invaluable additional structural information, enabling extremely complex and/or incommensurate structures to be identified and refined. Unfortunately, at present, much of the commercial software supplied with modern CCD instruments is of a 'black-box' variety written for ambient-pressure studies. As such, it is not designed for the peculiarities of high-pressure diffraction data, and knowledge and experience are often needed in order to coax a solution from the raw data. For example, the automatic 'harvesting' software typically used to locate single-crystal reflections can make no distinction between reflections from the sample and those originating from the diamonds and other pressure cell components. In many cases, therefore, the sample reflections must be identified by eye in the individual CCD images. CCD detectors also have other limitations for highpressure studies. The lack of any collimation on the large

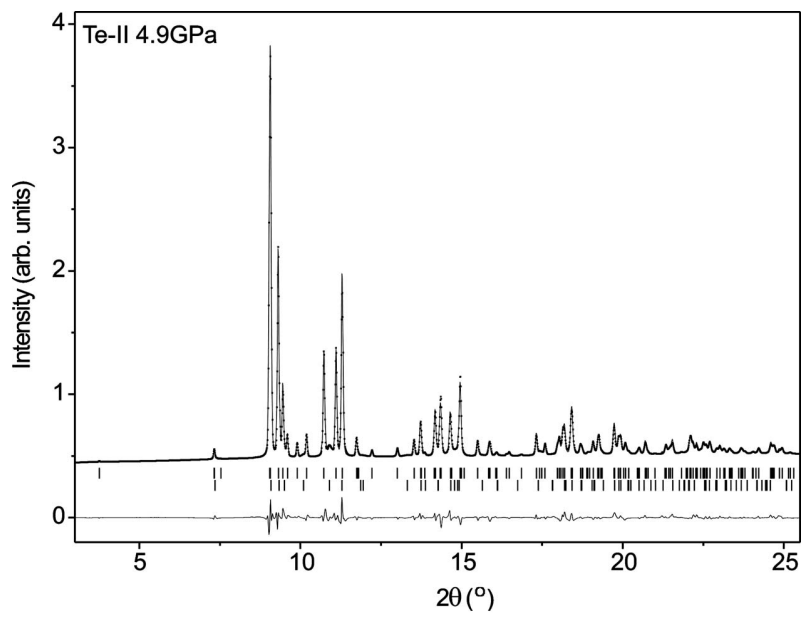

Figure 7

Rietveld refinement of triclinic Te-II at 4.9 GPa. The upper and lower tick marks show the calculated peak positions for Te-II and for a trace component of Te-III, respectively. The difference between the observed and calculated patterns is shown below the tick marks. Modified after Hejny \& McMahon (2004).

detector face leads to high structured backgrounds, which can make it difficult to obtain accurate intensities for very weak reflections. This problem can be overcome, however, by automatically masking out those regions of the CCD images that are affected by background scatter, and instructing the integration process to ignore them (Dawson et al., 2004). Secondly, during a typical data collection with a CCD, both strong and weak reflections are collected for the same amount of time. If one is particularly interested in extremely weak features, this may result in saturation of the strongest peaks. On station 9.8 at SRS, we typically collect two or more data sets from the sample at each pressure using different aluminium attenuators to increase the dynamic range of the detector. Finally, the CCD has no energy discrimination, and so diffraction features resulting from any $\lambda / 2$ radiation in the incident beam can be problematic, especially in determining space groups unambiguously. While the beamline $\mathrm{X}$-ray optics employed at synchrotrons normally eliminates harmonics, $\lambda / 2$ contamination is particularly important in data collected inhouse, where it results in a multitude of weak $\lambda / 2$ reflections from the diamond anvils that are of a similar intensity to the diffraction peaks from the sample. These can be eliminated, however, by reducing the X-ray generator voltage to below $35 \mathrm{keV}$. There may then be certain high-pressure problems where traditional four-circle diffractometers equipped with collimated energy-discriminating point detectors will prove to be a better choice of instrument, particularly if the intensities of very weak peaks are important. Of course, a combination of CCD and point-counter instruments, using the advantages of each to best effect, would be the best choice of all.

I gratefully acknowledge the contribution made to this work by my colleagues and co-workers, R. J. Nelmes, O. Degtyareva, C. Hejny, S. Rekhi, S. Falconi, L. F. Lundegard and J. S. Loveday. I also gratefully acknowledge the help of V. Petricek 
with using the JANA2000 Rietveld software. I thank M. A. Roberts and S. Teat of Daresbury Laboratory for assistance in setting up the 9.1 and 9.8 beamlines, respectively, and $\mathrm{M}$. Hanfland of the ESRF for setting up the ID09 beamline. This work was supported by grants from EPSRC, funding from CCLRC, and facilities provided by Daresbury Laboratory and the European Synchrotron Radiation Facility.

\section{References}

Belmonte, S. A. (1998). PhD thesis, The University of Edinburgh, UK.

Dawson, A., Allan, D. R., Parsons, S. \& Ruf, M. (2004). J. Appl. Cryst. 37, 410.

Degtyareva, O., McMahon, M. I. \& Nelmes, R. J. (2004a). Phys. Rev. $B, \mathbf{7 0}, 184119$.

Degtyareva, O., McMahon, M. I. \& Nelmes, R. J. (2004b). High Press. Res. 24, 319.

Hejny, C., Lundegaard, L. F., Falconi, S., McMahon M. I. \& Hanfland, M. (2005). Phys. Rev. B, 71, 020101.

Hejny, C. \& McMahon, M. I. (2003). Phys. Rev. Lett. 91, 215502.

Hejny, C. \& McMahon, M. I. (2004). Phys. Rev. B, 70, 184109.
McMahon, M. I., Bovornratanaraks, T., Allan, D. R., Belmonte, S. A. \& Nelmes, R. J. (2000). Phys. Rev. B, 61, 3135-3138.

McMahon, M. I., Degtyareva, O. \& Nelmes, R. J. (2000). Phys. Rev. Lett. 85, 4896-4899.

McMahon, M. I., Hejny, C., Loveday J. S., Lundegaard, L. F. \& Hanfland, M. (2004). Phys. Rev. B, 70, 054101.

McMahon, M. I. \& Nelmes, R. J. (2004). Z. Kristallogr. 219, 742.

McMahon, M. I., Rekhi, S. \& Nelmes, R. J. (2001). Phys. Rev. Lett. 87, 055501.

Nelmes, R. J., Allan, D. R., McMahon, M. I. \& Belmonte, S. A. (1999) Phys. Rev. Lett. 83, 4081-4084.

Nelmes, R. J. \& McMahon, M. I. (1994). J. Synchrotron Rad. 1, 69-73.

Nelmes, R. J. \& McMahon, M. I. (1998). Semicond. Semimet. 54, 145245.

Nelmes, R. J., McMahon, M. I., Loveday, J. S. \& Rekhi, S. (2002). Phys. Rev. Lett. 88, 155503.

Paszkowicz, W. (2002). Nucl. Instrum. Methods B, 198, 142-182.

Schwarz, U., Axelrud, L., Rosner, H., Ormeci, A. \& Grin, Yu. (2003) Phys. Rev. B, 67, 214101.

Shimomura, O., Takemura, K., Fujihisa, H., Fujii, Y., Ohishi, Y., Kikegawa, T., Amemiya, Y. \& Matsushita, T. (1992). Rev. Sci. Instrum. 63, 967-973.

Wright, N. G., Nelmes, R. J., Belmonte, S. A. \& McMahon, M. I. (1996). J. Synchrotron Rad. 3, 112-119. 